\title{
QCD finite T transition Comparison between Wilson and staggered results
}

\section{Yasumichi Aoki}

Brookhaven National Laboratory, Upton NY, USA

E-mail: yaoki@bnl.gov

\section{Zoltán Fodor}

Department of Physics, University of Wuppertal, Germany

Institute for Theoretical Physics, Eötvös University, Budapest, Hungary

E-mail: fodor@bodri.elte.hu

\section{Sándor D. Katz}

Department of Physics, University of Wuppertal, Germany

Institute for Theoretical Physics, Eötvös University, Budapest, Hungary

E-mail: katz@bodri.elte.hu

\section{Kálmán K. Szabó}

Department of Physics, University of Wuppertal, Germany

E-mail: szabokalgeneral.elte.hu

\section{Bálint C. Tóth*}

Institute for Theoretical Physics, Eötvös University, Budapest, Hungary

E-mail: tothbalinteszofi.elte.hu

A quantitative comparison between the finite temperature behaviour of the staggered and Wilson fermion formulations are performed. The comparison is based on a physical quantity that is expected to be quite sensitive to the fermionic features of the action. For that purpose we use the height of the peak for $d \chi_{s} / d T$, where $\chi_{s}$ is the quark number susceptibility.

The XXV International Symposium on Lattice Field Theory

July 30 - August 42007

Regensburg, Germany

\footnotetext{
*Speaker.
} 


\section{Introduction}

Staggered fermions are computationally faster than Wilson fermions, the discretization errors scale with $\mathscr{O}\left(a^{2}\right)$ and due to the well behaving spectrum of the Dirac operator, light quark masses nowadays even the physical ones - can be reached. Note, however, that a straightforward definition for staggered fermions exists only for $4,8, \ldots$ quark flavours. All works using 2 or $2+1$ flavours of staggered quarks use the fourth root (or square root) trick to have only one (or two) flavour(s). The action is defined by taking the fourth root of the fermion determinant. Since such a prescription is non-local at fixed lattice spacings, it is debated whether it is equivalent to a local continuum field theory or not (see e.g. [1-3] and references therein).

Large scale staggered studies are based on the expectation that in the continuum limit staggered and Wilson results agree. Clearly, at fixed, non-vanishing lattice spacings deviations are possible even if the continuum results are the same. Therefore, any analysis looking for the equivalence or non-equivalence between the staggered and Wilson formalism should fulfill two conditions. First of all, the analysis should be based on several lattice spacings and a controlled continuum extrapolation should be carried out. At least three different lattice spacings are needed, for which the asymptotic scaling behaviour can be already observed (both for staggered and for nonperturbatively clover-improved Wilson fermions one expects an $a^{2}$ scaling). Secondly, the physical quantity chosen as a basis of such comparison not only has to be well defined and relatively easily measurable, but also has to be sensitive to the dynamical fermion sector.

It is not so obvious how to find such a sensitive quantity. E.g. the masses of different hadrons provide well defined physical quantities, however, these are not sensitive enough to the dynamical fermion sector. Even the quenched calculations, where the dynamical fermions are completely omitted, provide the physical hadron masses with an error about or less then 10\% [4]. Finding measurable differences in the continuum limit of such quantities would require extremely high precision, thus, prohibitively large scale calculations.

Thermodynamic observables can be much more sensitive to the fermionic content of the theory. This fact is related to the singular/non-singular behaviour of the finite temperature QCD transition. E.g. on the one hand the quenched theory undergoes [5,6] a first order phase transition at non-vanishing temperatures $(\mathrm{T})$. For first order phase transitions different observables behave in a singular way (the latent heat is infinite or the temperature derivative of the renormalized Polyakov loop has a discontinuity). On the other hand the finite temperature transition of QCD with staggered fermions and physical quark masses has turned out to be a crossover [7]. As a consequence, none of the physical quantities as the function of the temperature are infinite or discontinuous, at the most they only undergo a rapid change within a narrow temperature range. Clearly, the height of such a peak is expected to be quite sensitive to the details of the fermionic properties of the action. As we increase or decrease the quark masses the peak turns out to be more and more singular and after a while a second order then a first order phase transition region is reached. Thus, the fermionic content (quenched or unquenched with physical or non-physical quark masses) manifests itself in a very pronounced way.

Choosing a physical quantity that undergoes a rapid change and finding the maximum of its derivative with respect to the temperature may provide a quantity sensitive to the finite temperature behaviour of the system. 
The aim of this study is to provide a quantitative comparison between the behaviour of the staggered and Wilson fermion formulations. We attempt to perform an analysis at two different lattice spacings (thus no conclusive continuum extrapolation can be done yet). The observable we have chosen is related to the transition at non-vanishing temperatures and expected to be sensitive to the fermionic properties of the theory.

One physical quantity that undergoes a rapid change around the transition temperature is the quark number susceptibility, which is defined via [8]

$$
\frac{\chi_{s}}{T^{2}}=\left.\frac{1}{T V} \frac{\partial^{2} \log Z}{\partial \mu^{2}}\right|_{\mu=0},
$$

where $\mu$ is the quark chemical potential. The quark number susceptibility can be directly measured, and it automatically has the correct continuum limit without the need for renormalization. In addition, the maximum of its derivative with respect to the temperature, that is, the rate at which the susceptibility changes during the transition, is sensitive to the dynamical fermion sector. These properties make the quark number susceptibility a good candidate for the quantity that a comparison of the Wilson and staggered fermion formulations should be based on. Since the rooting procedure of the determinant is less transparent (might be more problematic) for odd number of flavours we use three flavours. (The one flavour theory, where there is no chiral symmetry breaking [9], is not suitable for our purposes.)

\section{Action parameters}

The three flavours were degenerate for both Wilson and staggered calculations, and the gauge action used was the Symanzik tree-level improved gauge action [10]. In both cases the lattices sizes were $32^{3} \times 8$ and $32^{3} \times 10$, and the configurations were generated using the Rational Hybrid Monte-Carlo algorithm [11].

Wilson calculations: Three steps of stout smearing [12] with smearing parameter $\rho=0.1$ were used. The gauge coupling constant was in the range $\beta=3.2-3.7$. In addition the femionic sector was clover improved [13] with a tree level clover coefficient $c=1.0$. Note, that for this type of smeared fermions the tree level clover coefficient essentially leads to an $\mathscr{O}(a)$ improved action [14].

Staggered calculations: Two steps of stout smearing with smearing parameter $\rho=0.15$ was used [15], and the gauge coupling constant was in the range $\beta=3.5-4.0$. This staggered action within this lattice spacing range was shown to be in the scaling regime [16].

\section{Setting the scale}

To be able to make sure that the staggered and Wilson calculations are performed at the same set of physical parameters, the line of constant physics was defined by fixing the ratio of the pseudoscalar and the vector meson masses $m_{\mathrm{PS}} / m_{\mathrm{V}}$.

If the finite temperature behaviour of the Wilson and the staggered formulations are different, the difference is likely to be most apparent at small quark mass parameters. For small quark masses we are closer and closer to the first order phase transition region, thus all the differences are easier 

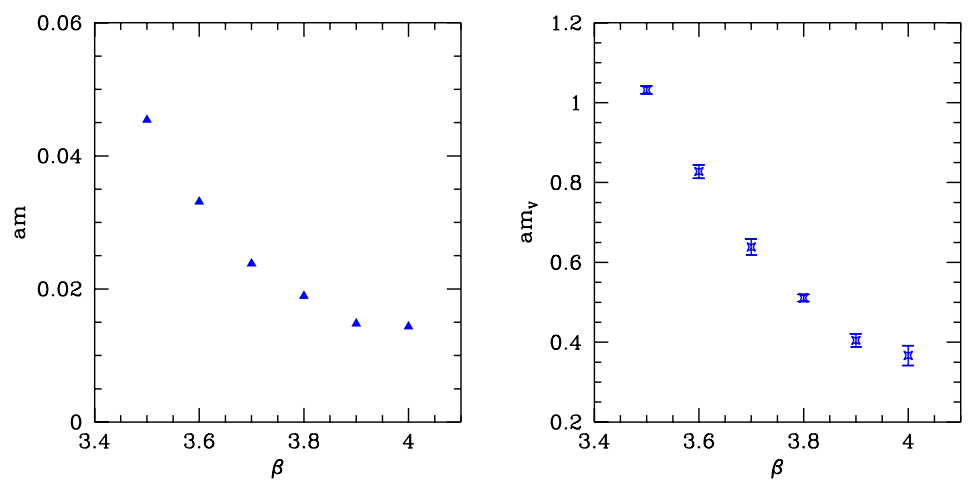

Figure 1: The scale for the staggered calculations: the bare staggered quark mass (left panel) and the vector meson mass (right panel) as a function of the gauge coupling constant.
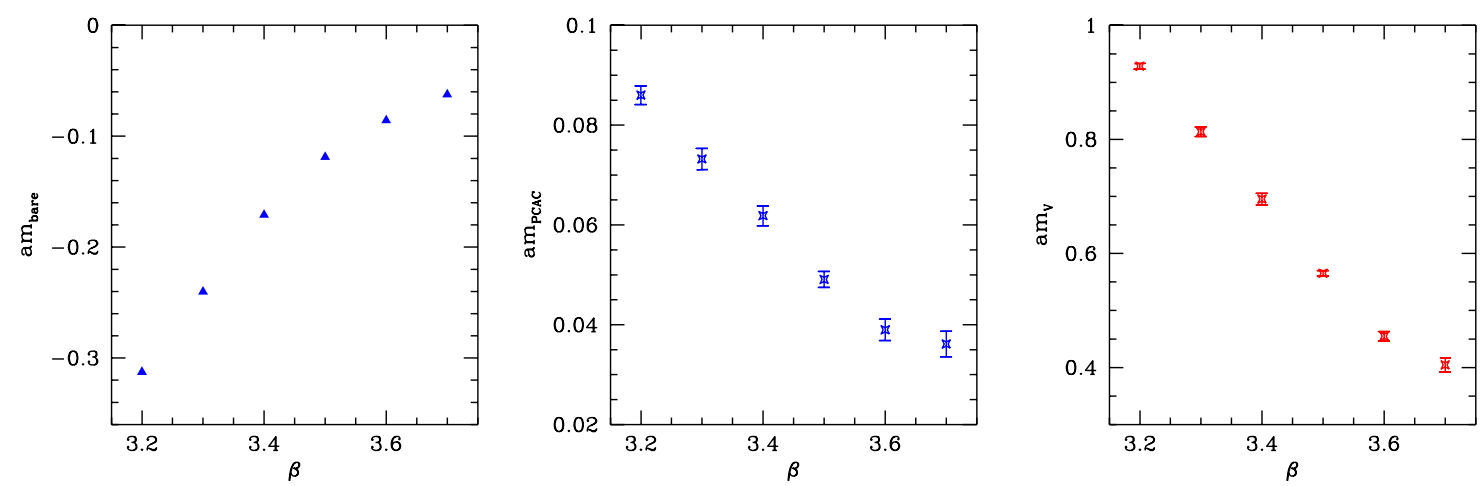

Figure 2: The scale for the Wilson calculations: the bare Wilson quark mass (left panel), the PCAC quark mass (middle panel) and the vector meson mass (right panel) as a function of the gauge coupling constant.

to see in the height of the peak of our observable. Decreasing the quark mass, however, causes the computational costs to rise. Therefore, one needs to find a compromise between making the comparison more sensitive by lowering the quark mass (or in other words lowering $m_{\mathrm{PS}} / m_{\mathrm{V}}$ ) and keeping the computational costs reasonable.

As a compromise we have chosen $m_{\mathrm{PS}} / m_{\mathrm{V}}=0.5$, which sets the quark mass about $m_{s} / 3$, where $m_{s}$ is the physical strange quark mass. This relationship completely defines the line of constant physics. In three flavour lattice QCD we have two parameters. One of them is the quark mass which is essentially set by the relationship between the pseudoscalar and vector mass ratio. The other one is the lattice spacing, which is dominantly given by the gauge coupling.

The bare quark mass and the vector meson mass corresponding to the different gauge couplig values for the staggered case are shown in Figure 1. For the Wilson calculations, the bare quark mass, the current algebra quark mass and the vector meson mass are shown in Figure 2 . 

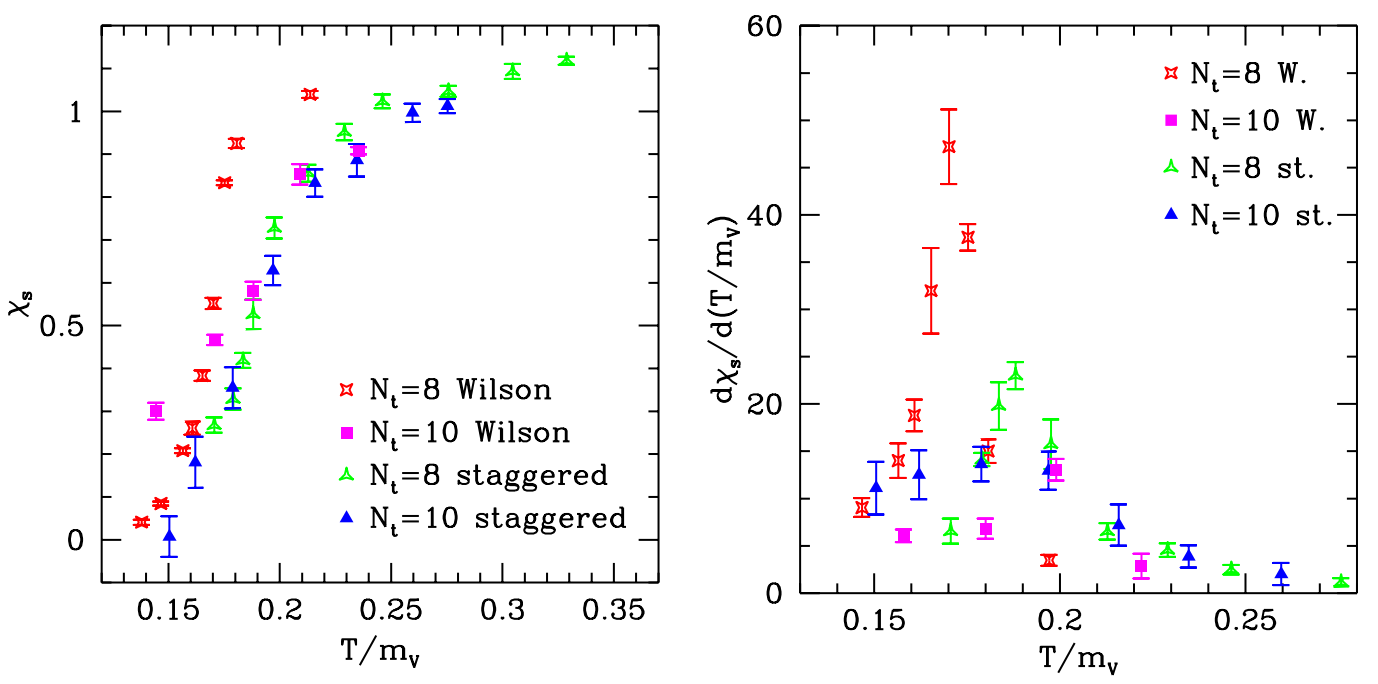

Figure 3: The quark number susceptibility (left panel) and its derivative (right panel) as a function of the temperature normalized with the vector meson mass.

\section{Results}

To be able to compare the staggered and Wilson results the temperature was made dimensionless by dividing by the vector meson mass. The quark number susceptibilities as a function of $T / m_{\mathrm{V}}$ are shown in the left panel of Figure 3 . The derivative of the susceptibility was obtained by fitting cubic polynomials to the susceptibility points, then taking the derivative of the polynomial. The slight change due to the variation of the fitting range is taken as a systematic error. These derivatives are shown in the right panel of Figure 3 .

\section{Conclusions}

Calculating the maximum of the derivative of the quark number susceptibility with respect to the temperature yields a sensitive quantity, based on which the finite temperature behaviour of the Wilson and the staggered fermion formulations can be compared. We have performed calculations using lattices with temporal extensions $N_{t}=8$ and 10. At $N_{t}=8$ the maximum of the dervative in the Wilson case is a factor of 2 higher than the staggered result, whereas at $N_{t}=10$ the heights of the peaks get closer to one another. In order to be conclusive one needs to get one or more steps closer to the continuum limit by performing calculations using $N_{t}=12$ or even finer lattices.

\section{Acknowledgements}

This research was partially supported by OTKA Hungarian Science Grants No. T34980, T37615, M37071, T032501, AT049652, EU Grant I3HP and by DFG German Research Grant No. FO 502/1-1. The computations were carried out on the 370 processor PC cluster of Eötvös University, 
on the 1024 processor PC cluster of Wuppertal University and on the Blue Gene/L in Research Centre Jülich. We used a modified version of the publicly available MILC code [17] with next-neighbor communication architecture for PC-clusters [18].

\section{References}

[1] C. Bernard, M. Golterman and Y. Shamir, Observations on staggered fermions at non-zero lattice spacing, Phys. Rev. D73 (2006) 114511 [hep-lat/060401]].

[2] M. Creutz, Why rooting fails, PoS(LATTICE 2007)007 (2007) [arXiv:0708.1295 [hep-lat].

[3] A. Kronfeld, Lattice QCD with staggered quarks: Why, where, and how (not), PoS(LATTICE 2007)016 (2007).

[4] CP-PACS Collaboration, S. Aoki et al., Light hadron spectrum and quark masses from quenched lattice QCD, Phys. Rev. D67 (2003) 034503 [hep-lat/0206009].

[5] T. Celik, J. Engels and H. Satz, The order of the deconfinement transition in SU(3) Yang-Mills theory, Phys. Lett. B125 (1983) 411.

[6] M. Fukugita, M. Okawa and A. Ukawa, Order of the deconfining phase transition in SU(3) lattice gauge theory, Phys. Rev. Lett. 63 (1989) 1768.

[7] Y. Aoki, G. Endrodi, Z. Fodor, S. D. Katz and K. K. Szabo, The order of the quantum chromodynamics transition predicted by the standard model of particle physics, Nature $\mathbf{4 4 3}$ (2006) 675-678 [hep-lat/0611014].

[8] MILC Collaboration, C. Bernard et. al., QCD thermodynamics with three flavors of improved staggered quarks, Phys. Rev. D71 (2005) 034504 hep-lat/0405029].

[9] M. Creutz, One flavor QCD, Annals Phys. 322 (2007) 1518-1540 hep-th/0609187.

[10] K. Symanzik, Continuum limit and improved action in lattice theories. 1. Principles and $\phi^{4}$ theory, Nucl. Phys. B226 (1983) 187.

[11] M. A. Clark and A. D. Kennedy, The RHMC algorithm for 2 flavors of dynamical staggered fermions, Nucl. Phys. Proc. Suppl. 129 (2004) 850-852 [hep-lat/0309084].

[12] C. Morningstar and M. J. Peardon, Analytic smearing of $S U(3)$ link variables in lattice QCD, Phys. Rev. D69 (2004) 054501 [hep-lat/0311018].

[13] B. Sheikholeslami and R. Wohlert, Improved continuum limit lattice action for QCD with Wilson fermions, Nucl. Phys. B259 (1985) 572.

[14] R. Hoffmann, A. Hasenfratz and S. Schaefer, Non-perturbative improvement of nHYP smeared Wilson fermions, PoS(LATTICE 2007)104 (2007) [arXiv:0710.0471 [hep-lat]].

[15] Y. Aoki, Z. Fodor, S. D. Katz and K. K. Szabo, The equation of state in lattice QCD: With physical quark masses towards the continuum limit, JHEP 01 (2006) 089 [hep-lat/0510084].

[16] Y. Aoki, Z. Fodor, S. D. Katz and K. K. Szabo, The QCD transition temperature: Results with physical masses in the continuum limit, Phys. Lett. B643 (2006) 46-54 [hep-lat/0609068.

[17] MILC Collaboration, Public lattice gauge theory code, online at http://physics.indiana.edu/ $/$ sg/milc.html.

[18] Z. Fodor, S. D. Katz and G. Papp, Better than \$1/Mflops sustained: A scalable PC-based parallel computer for lattice $Q C D$, Comput. Phys. Commun. 152 (2003) 121-134 [hep-lat/0202030]. 